\title{
Recruitment Strategies and Rates of a Multi-site Behavioral Intervention for Adolescents and Young adults with Cancer
}

\author{
Verna Hendricks-Ferguson, PhD, RN [Professor], \\ Goldfarb School of Nursing, 4483 Duncan St., St. Louis, MO 63110, (C) 618-558-9759
}

Brooke Cherven, RN, MPH, CPON [Clinical Research Nurse],

Aflac Cancer Center and Blood Disorders Service, Children's Healthcare of Atlanta, 2015 Uppergate Drive 4th FI, Atlanta, GA 30306, (P) 404-785-4226

Debra S. Burns, PhD, MT-BC [Associate Professor],

Coordinator, Music Therapy Programs, Dept of Music and Arts Technology, Purdue School of Engineering and Technology @ IUPUI, 535 W. Michigan St., IT 379, Indianapolis, IN 46202, (P) 317-278-2014

Sharron L. Docherty, PhD, CPNP (AC/PC) [Associate Professor], Duke University School of Nursing, DUMC 3322, 307 Trent Drive, Room 359, Durham, NC, 27710, (P) 919-668-3836

Celeste R. Phillips-Salimi, PhD, RN, CPON [Assistant Professor], University of Kentucky, College of Nursing, 449 CON Building, Lexington, KY 40536, (P) 317-442-2973

Lona Roll, MSN, RN [Consultant], The University of Texas Health Science Center at San Antonio, Department of Pediatrics, 7703 Floyd Curl Drive, San Antonio, Texas 78229-3900, (P) 210 394-2611

Kristin A. Stegenga, RN, PhD, CPON [Nurse Researcher], Children's Mercy Hospital, 2401 Gillham Road, Kansas City, MO 64108, (P) 816-234-3292

Joan E. Haase, RN, PhD, FAAN [Holmquist Professor in Pediatric Oncology Nursing], and Indiana University School of Nursing, 1111 Middle Drive, Office NU 492B, Indianapolis, IN 46202, (P) 317-439-2380

Molly Donovan Stickler, MPH [Research Associate, Project Manager] Indiana University School of Nursing, 1111 Middle Drive Mailbox: NU 340, Office: NU B10-J, Indianapolis, IN 46202, (P) 317-278-7749

Verna Hendricks-Ferguson: verna@charter.net; Brooke Cherven: Brooke.Cherven@choa.org; Debra S. Burns: desburns@iupui.edu; Sharron L. Docherty: Sharron.docherty@duke.edu; Celeste R. Phillips-Salimi: cphillipssalimi@uky.edu; Lona Roll: ljr5055@gmail.com; Kristin A. Stegenga: kstegenga@cmh.edu; Joan E. Haase: johaase@iupui.edu; Molly Donovan Stickler: madonova@iupui.edu

\section{Keywords}

Recruitment rates; research; adolescents with cancer

\footnotetext{
(C) 2012 National Association of Pediatric Nurse Associates and Practitioners. Published by Mosby, Inc. All rights reserved. Publisher's Disclaimer: This is a PDF file of an unedited manuscript that has been accepted for publication. As a service to our customers we are providing this early version of the manuscript. The manuscript will undergo copyediting, typesetting, and review of the resulting proof before it is published in its final citable form. Please note that during the production process errors may be discovered which could affect the content, and all legal disclaimers that apply to the journal pertain.
} 


\section{INTRODUCTION}

Researchers, clinicians and national organizations such as the National Institutes of Health continue to emphasize the need to design studies that maximize the translation of results to patient care (Kleinpell, 2008). Critical to the success of all research protocols and ultimately to the translation of research findings into practice is the inclusion of a carefully developed, implemented, and evaluated recruitment plan. A well-developed plan should enhance the potential for successful accrual of the desired number of participants and for sample representativeness of the target population (May et al., 2007).

Meeting recruitment goals can be challenging with some target populations, such as adolescents and young adults (AYA). The unique developmental needs and changes that AYA specifically experience can compound the challenges to recruitment and retention. Only recently have researchers described recruitment processes specific to AYA with cancer. However, no theoretically or empirically based guidelines and strategies are available for investigators to consider when developing and implementing a recruitment plan for AYA with cancer (Gattuso, Hinds, Tong, \& Srivastava, 2006).

To provide a model for AYA recruitment, the purpose of this paper is to describe our recruitment strategies, participants' reasons for consent or refusal, and the final recruitment rates of AYA with cancer into a research study called Stories and Music for Adolescent/ Young Adult Resilience during Transplant" (SMART). The SMART study was a joint National Institute of Nursing Research (R01NR008583) and National Cancer Institute (U10CA098543; U10CA095861) funded cooperative group study (Children's Oncology Group ANUR0631) thatevaluated the efficacy of a behavioral intervention (specifically, a therapeutic music video intervention compared to a low-dose audio-book control group) among AYA with cancer, ages 11 to 24 years who were undergoing a hematopoietic stem cell transplant (HSCT) for an oncology condition. The recruitment strategies and outcomes reported in this article may help investigators enhance recruitment with other AYA populations.

\section{BACKGROUND}

\section{AYA Recruitment Rates}

Because few research reports include recruitment rates and reasons for refusal specific to the AYA population, investigators have little guidance when developing recruitment plans. Studies including AYA as a sub-group of a larger sample of children or adults have not reported the AYA recruitment rates apart from their older and younger participants (Bleyer, Budd, \& Montello, 2006; Cripe, 2004).

In recent years, the Children's Oncology Group (COG), a cooperative clinical trials group funded by the National Cancer Institute (NCI), has increased its focus on providing opportunities to recruit children and AYA participants to randomized clinical trials (RCTs) across clinical sites in North America, Australia, New Zealand and parts of Europe. To date, recruitment rates to clinical RCTs for eligible children (i.e., aged less than 15 years of age) are 40 to $70 \%$ (Burke, Albritton, \& Marina, 2007). In contrast, fewer than 10\% of eligible AYA between 15 and 24 years of age are typically recruited to RCTs (Burke et al., 2007). Therefore, in recent years COG has increased its efforts to focus on increasing the recruitment rates of eligible AYA to clinical RCTs.

Factors related to successful recruitment strategies and rates for AYA with cancer are not commonly described in published clinical research studies. Reports of AYA recruitment and refusal rates have been limited to psychosocial/behavioral health studies (Broome \& 
Richards, 2003; Hinds et al., 1997; Hinds et al., 2001; Hinds et al., 2000; Hinds et al., 2002; Hudson et al., 2002; Kazak et al., 2004; Knox \& Burkhart, 2007; Pasternak, Geller, Parrish, $\&$ Cheng, 2007). A majority of studies reporting recruitment rates for AYA with cancer have been descriptive, and similar to clinical trials, often grouped within a sample of participants who were younger or older than an AYA (aged 11 to 24 years). Descriptions of specific AYA recruitment strategies and reasons for declining participation are still missing from the literature. (Malbasa, Kodish, \& Santacrose, 2007).

\section{Factors Influencing AYA Recruitment Rates}

Although investigators have proposed general factors that influence recruitment rates, there is scant information reported in the literature related to AYA-specific factors that may impact recruitment of AYA with cancer to research studies. Factors related to AYA recruitment can be postulated by developmental considerations, AYA and parent perspectives, healthcare system factors and research design issues.

Developmental Considerations-AYA function individually in an integrated system, specifically from internal (i.e., physiological, cognitive, emotional) and external (e.g., family, peer group, culture) subsystems that contribute to their development (Jones \& Broome, 2001; Magnusson \& Cairns, 1996; Miles \& Holditch-Davis, 2003). Consideration of these unique subsystems is important because of their potential impact upon AYA's decisions to participate in research. For example, a 14-year-old adolescent may provide different reasons for declining participation than a 17-year-old adolescent.

In general, adolescent cognition shifts dramatically from a literal focus on the present in early adolescence to a more abstract and future-oriented focus in late adolescence (Rice \& Dolgin, 2005). Emotional maturity varies widely among AYA and may affect their needs and coping responses. Also, a cancer diagnosis can sometimes result in AYA returning to earlier levels of dependence on parents or other family members (Broome \& Richards, 2003).

AYA and Parent Perspectives-Another challenge to recruitment of AYA into studies is the consenting procedures related to the AYA's age range (assent versus consent) and parents' right to approve AYA participation. Thus, the decision to participate may include decisions made by AYA, parents, or both. Though AYA generally trust their parents' decision regarding their involvement in research, the AYA may not understand that their parents' consent is still required (Broome \& Richards, 2003; Knox \& Burkhart, 2007; Pasternak et al., 2007).

Reported reasons for parents to decline their AYA's involvement in studies have included: (a) risk of randomization to an undesired treatment group (Bleyer, 2006; Hinds, 2000); (b) inconvenience of participation (Harth, 1995; Hayman, Taylor, Peart, Galland, \& Sayers, 2001; Hinds et al., 2002; Kazak et al., 2004); (c) safety concerns (Caldwell, Butow, \& Craig, 2003; Harth, 1995; Hayman et al., 2001); and (d) potential side effects of proposed treatments (Bowman, Vidsten, Kogner, \& Samuelsson, 2004; Hendricks-Ferguson, 2006). Also, AYA decline participation due to: (a) lack of interest or perceived benefit (Gattuso et al., 2006; Hinds et al., 2002; Ruffin \& Baron, 2000); (b) symptom distress (Crowley \& Casarett, 2003; Gattuso et al., 2006; Hendricks-Ferguson, 2006; Hinds et al., 2000; Kyngas et al., 2001); (c) engagement in other activities, such as homework or video games (WardSmith, Hamlin, Bartholomew, \& Stegenga, 2007); and (d) not wanting to revisit their cancer experience (Kazak et al., 2004). 
Healthcare System Factors-Research has suggested that AYA are more likely to participate in research if they are referred by their own health care provider (e.g., physician or nurse) (Breland-Noble, 2006; Gooch, 2000; May et al., 2007; Seibold-Simpson \& Morrison-Beedy, 2010; Yancey, Ortega, \& Kumanyika, 2006). AYA are not necessarily more likely to participate in research simply because their treatment setting is located in an academic medical center. Furthermore, receiving medical treatment at an academic medical center does not guarantee eligibility of AYA with cancer for research because most clinical trials do not focus on cancers specific to AYA. These factors also contribute to limited evidence about how to plan effective strategies to recruit AYA with cancer to clinical RCTs (Burke et al., 2007; Ferrari \& Bleyer, 2007).

Research Design Issues-While study designs need to be rigorous to obtain valid results, certain design considerations can create barriers to recruitment. Stringent inclusion and exclusion criteria decrease the pool of potential participants. Randomization to a treatment group presents a particular challenge to recruitment because AYA often prefer to make a choice rather than to be assigned to the available group options (Broome \& Richards, 2003). This preference may also increase the risk of eligible AYA refusing to participant in the study while it may also increase the need for a larger pool of eligible participants to obtain a sample with desired study characteristics. In addition, investigators must consider AYA attrition, which can be influenced by illness acuity, developmental stage, and mortality risk (Broome \& Richards, 2003).

In summary, there are multiple barriers to the recruitment of AYA into RCTs. This is a challenge to researchers who are designing research studies that include AYA, especially recruitment. To ensure translation of research results involving AYA into clinical practice, recruitment strategies must be tailored to accommodate the needs of this often overlooked population. The processes for planning, implementing, and monitoring recruitment for the SMART study provide examples of successful strategies and barriers to recruitment of AYA.

\section{PROCESSES OF RECRUITMENT OF AYA TO RCT}

The SMART study was conducted at 6 pediatric and 3 adult hospitals across 6 medical centers throughout the Midwest and southern portions of the United States. Institutional Review Board approval was obtained at each study site and the enrolled participants provided informed consent. The primary aim of the study was to evaluate the efficacy of a music therapy intervention to influence symptom distress, coping, resilience and quality of life in AYA with cancer.

The targeted sample was AYA, with equal representation from both genders and 3 age groups (11-13, 14-17, and 18-24 years old). Inclusion criteria were: (a) AYA between 11 and 24 years of age, (b) current treatment at a COG member institution with either an allogeneic or autologous hematopoietic stem cell transplant (HSCT) for cancer with a myeloablative regimen and (c) ability to read and speak English. Exclusion criteria were: (a) a diagnosis of cancer not usually occurring in childhood or AYA populations (e.g., breast or prostate cancer); (b) involvement of active central nervous system tumors or other cognitive impairments that would hinder AYA's participation in the intervention and/or completion of the questionnaires and (c) AYA who were married and/or had children. The last exclusion criterion was not initially identified but emerged as important after recruiting efforts demonstrated a distinct subset in this category. It was determined that AYA who were already married and/or having a child were developmentally distinct from the targeted population and the measures pilot-tested for this study had not been tested in this sub-group of AYA. 


\section{Recruitment Plan for AYA}

Strategies for Site Selection-We carefully considered site characteristics that we hypothesized would be conducive to successful AYA recruitment to a multi-site RCT. For the SMART study, we used specific criteria to identify potential sites, including: (a) COG member institution, including Clinical Cancer Oncology Program (CCOP) institutions, with adequate numbers of AYA patients undergoing HSCT to contribute to the total sample with respect to gender, age, and ethnic diversity; (b) a well-established, working relationship between the site principal investigator (PI) (either nurse scientist or advanced practice nurse) and the transplant unit staff; (c) explicit HSCT physician support; (d) qualified interveners (i.e., board-certified music therapists) available either in the hospital or vicinity; (e) reasonable facility and administration (indirect) costs within the study grant budget and (f) ability to provide personnel support for data collection. In addition, less objective, yet essential site qualities (e.g., responsiveness to requests for information and meetings) and a strong verbalized desire and commitment to participate were considered.

Investigators evaluated potential participant availability at each site by requesting data on the number of AYA in the 11- to 24-year age range who had received an HSCT in the previous 2 years. This enabled the calculation of the number of sites needed to complete the study in the proposed time frame, allowing for a conservative recruitment rate of $65 \%$ and a $50 \%$ attrition rate since AYA undergoing HSCT are known to be a high acuity, high risk population.

Training for Successful Recruitment-We considered training to be of utmost importance to assure adequate knowledge of the study and to provide a consistent and effective approach to all aspects, including recruitment. For the SMART study, individual site personnel participated in $2 \frac{1}{2}$ days of training involving: (a) discussion of the purpose of the study and key components of the two theoretical frameworks on which the study was based, the Resilience in Illness Model (formerly the Adolescent Resilience Model) (Haase, 2004) and the Robb Contextual Support Model (Robb, 2000); (b) description of roles, responsibilities and expectations utilizing role-specific study materials; (c) recruitment, intervention and/or evaluation activities and (d) attainment of personnel commitment to the study through role-specific signed contracts. Specific teaching strategies used during recruitment training included discussions and role-playing scenarios to practice developmentally appropriate communication and to facilitate sensitivity when talking with AYA and family members. At this training, strategies were provided to help study personnel identify and address AYA concerns and appeal to their natural desire for enjoyment and distraction during their HCST. Team-building activities were also part of the curriculum to foster perception of study personnel as members of a team doing important work.

General Recruitment Plan-A comprehensive and theoretically driven recruitment plan was developed to delineate the following recruitment tasks that each site needed to accomplish: (a) identifying participants who met inclusion criteria; (b) identifying the best person and optimal time to approach potential participants; (c) incorporating recruitment strategies that accommodated adolescent developmental tasks (e.g., autonomy) and interests and (d) tracking and documenting potential participants. The plan included timely communication expectations between the site-team members (i.e., project manager, site PI) regarding recruitment. Around these general tasks, site-team members tailored the plan to their unique locations to optimize success. The Health Insurance Portability and Accountability Act (HIPAA) regulations were a key factor in planning. Depending on study personnel affiliations and roles and on whether or not a HIPAA waiver was required (i.e., if study personnel were employed outside the institution), the site-project manager and/or principal investigator (PI) regularly attended HSCT meetings to help identify potential 
participants or to receive information regarding potentially eligible participants, and a plan was made to approach potential participants. This ensured regular communication between the transplant team and the study team while adhering to HIPAA regulations.

The site-specific plans for presenting the study to eligible participants varied, based on typical working arrangements. Every effort was made to approach the AYA and parent(s) prior to hospital admission for transplant before the AYA were experiencing high levels of symptom distress. Generally, initial contact was made by a healthcare team member (i.e., physician or nurse coordinator) who had an established relationship with the eligible AYA. This team member briefly introduced the study using a colorful, AYA-friendly brochure and obtained permission to share specific contact information with study staff. This approach was believed to foster trust and enthusiasm and ultimately to facilitate study enrollment. Subsequently, the project manager or site PI contacted the AYA and parent(s) to discuss the study in more detail and obtain consent/assent.

Tracking Procedures for Enrollment and Refusal-We carefully considered a recruitment plan for our study to include specific mechanisms for tracking participantrelated information. All potential SMART participants were tracked with a de-identified data spreadsheet maintained on a secure web-based program accessible at all sites by appropriate personnel. The spreadsheet assistedin: (a) monitoring the numbers of eligible participants, (b) tracking study accrual/refusal rates and reasons, and (c) providing a tool for across-site discussion of recruiting strategies. During bi-weekly conference calls, site and core PIs and project managers reviewed the spreadsheet, addressed problems regarding recruitment challenges and identified successful strategies.

Ethical Considerations-Because of unique age range and developmental characteristics, recruitment of AYA required careful consideration of the ethical principles in the conduct of research, such as: (a) decision-making autonomy regarding study participation for AYA younger than 18 years of age and (b) privacy and respect during presentation of the study to AYA and parents. The expectation was that the AYA's decision to participate should be autonomous, and consent/assent would be obtained only after it was clear that the study purpose and requirements were understood by the AYA and parents as appropriate. All AYA were assured that their participation was voluntary and any decision to decline or withdraw from the study would not affect their care. In addition, privacy and respect for the AYA were taken into consideration by recruiters. Specifically, AYA and parents were invited to a private room when introduced to the study and when asked about their preferences regarding receiving information about the study.

\section{RECRUITMENT RESULTS FROM THE SMART STUDY}

AYA Recruitment-The SMART study closed for accrual in 2010. Enrollment was extended a few months beyond the initial planned 4-year time period to achieve a sufficient sample size to address the study aims. Using tailored recruitment processes to address the key factors listed above, an overall recruitment rate of 50\% was achieved, which is within the range of previous studies (i.e., range of 47 to $65 \%$ ). Figure 1 is a CONSORT diagram of recruitment and study participation. Enrollment information for participants according to recruitment by age and gender are presented in Table 1. Among the 118 enrolled AYA, more males $(n=67)$ were enrolled than girls $(n=51)$. However, a higher percentage of females enrolled in each of the 3 targeted-age groups compared to males. Although males had a lower enrollment rate according to age, the high number of available eligible males resulted in more male participants than females. 
Reasons for AYA to Enroll or Decline Participation-AYA usually spontaneously offered their reasons for consenting or declining participation. When AYA declined to participate but did not volunteer a reason, the recruiter invited them to share their reasons.

AYA offered several reasons for participating. Some were motivated by altruism and a desire to help patients who would go through similar struggles. This rationale is consistent with the tenet of altruism that is recognized in adolescent developmental theory as reported in the literature (Rice \& Dolgin, 2005). Other AYA looked forward to using the study sessions and activities as a form of distraction while hospitalized. Some participants found the study activities appealing, describing themselves as very musical or loving books and reading. Health care providers at each site maintained ongoing communication to encourage AYA about the benefits of participation. The relationships that were established with the research team members who introduced the study, such as the HSCT nurse coordinators and transplant physicians, may have influenced some AYA's decision. It is difficult to discern if encouragement from their parents to participate may have also been an influencing factor.

AYA offered a wide range of reasons for declining participation. These included: (a) no desire for extra commitments; (b) interference of the study with other activities or learning experiences; (c) commitments outside of HSCT, such as jobs or online courses; (d) competing RCT studies offering incentives; (e) unwillingness to be randomized with preferences for either the therapeutic music video (TMV) intervention group or the bookson-tape group and (f) emotional or symptom distress precluding ability to participate.

As a qualitative example for declining, one of the AYA stated: "This is all pretty new to me and I just don't want to commit to anything. If anything, I guess I might do that other study where they pay me. That's the only thing that stands out." Another said that his music teacher planned to teach him banjo during his hospital admission, and he wanted to focus on that. Still another stated that she would not want to do the books-on-tape group because she didn't like any of the books on the list.

\section{Discussion}

The factors influencing recruitment of AYA identified in the literature were supported by recruitment experiences with the SMART study. These factors and additional strategies to improve recruitment of AYA are discussed below. Although several strategies were identified as important to the successful recruitment of a representative sample of ethnic and racial minorities, it is beyond the scope of this paper to discuss the ways cultural and racial perspectives also may affect recruitment of AYA. In a future manuscript, we plan to report findings regarding cultural and ethnic factors that also may have affected our recruitment of AYA.

Participant and Developmental Characteristics-Consistent with the literature, the SMART study had higher recruitment rates for females in general and younger female AYA (Seibold-Simpson, 2010). The more successful recruitment of young females supports an assertion of Gilligan that adolescent females place more emphasis on relationships (Gilligan, 1982); accordingly, the young adolescent females in SMART may have valued the opportunity to work closely with the interventionist. The gender outcome is consistent with a recent study of adolescents with HIV (Seibold-Simpson, 2010). The more successful recruitment of young adolescent females is supported in the developmental literature (Nelson, 2003; Carlo, 2003), suggesting that younger females often have a strong desire to please others. Therefore, younger female adolescents may be inclined to participate in research studies. 
The fact that male AYA represented the highest number of eligible patients is likely related to the inclusion of a study site that specializes in the treatment of testicular germ cell cancers, a common cancer in the young-adult age group. The lower recruitment rate among male AYA is consistent with research findings that male AYA are less likely to participate in randomized trials (Gattuso et al., 2006; Hinds, Quargnenti, \& Madison, 1995). Careful initial planning and monitoring of recruitment rates for specific age and gender groups is essential. We addressed an early indication of a higher refusal rate in young adult males and their expressed lack of interest in the books on the list by adding a book more appealing to this group. Qualitative studies would be useful to further explore the reasons why male AYA with cancer may be more likely to decline enrollment in RTCs.

Another developmental lesson learned was some older AYA's declined to participate because they planned ahead to focus on college and career goals during hospitalization for their SCT. This finding supports Erikson's theory of psychosocial development (Erikson, 1963), which asserts that older adolescents tend to be focused on their future goals. Consideration of the future orientation of older AYA is important for investigators to consider when planning timeframes for approaching older AYA for recruitment.

The positive stories of AYA experiences shared across sites enabled SMART personnel to describe participation as a potentially fun, creative way to pass time during HSCT and something over which AYA had control during a time when many daily activities were out of their control. The literature supports recruiters sharing the positive experiences of other participants as a key factor in successful recruitment. (Gattuso et al., 2006; Hinds et al., 1995).

Although legal guidelines direct that parents must consent for AYA younger than 18 years of age, we found that the nature of the relationship between the AYA and their parents, regardless of age, was important in their decision to participate in our study. When AYA had a close and mutually respectful relationship with their parents, the decision to participate was shared. Also, when shared decision-making occurred, the AYA and parents seemed more committed to the study. This suggests that fostering shared decision making about participation in research may result in more successful recruitment and retention (Broome, 2003). However, future research is needed focusing on AYA recruitment rates in relation to the nature of the AYA-parent relationship.

Design features-The timing of the study introduction is critical for successful recruitment. Some of the HSCT patients in SMART declined participation because of symptom distress. We found it was very important to approach potential participants as soon as possible after their HSCT was scheduled. Also, potential participants who have just completed the in-depth discussion of potential side effects of transplant may not be as receptive to information as they would be if they were approached at a different time in their pre-transplant work-up.

When the study team encountered AYA who seemed hesitant to participate in the SMART study, the site project manager or site PI encouraged the AYA to ask questions, which then gave opportunity for clarification, often alleviating concerns and resulting in interest in the study and consent to participate. This strategy proved to be successful and supports the importance of open communication to the successful recruitment of AYA to RCTs (Broome, 2003).

Early identification of recruitment patterns may allow changes in the study design to facilitate recruitment strategies. Revisions to the design should be made with caution, however, because they may adversely affect the rate of recruitment. For example, our late 
decision to exclude AYA who were married or had children reduced our recruitment rates more than expected, resulting in a longer accrual time in an attempt to reach the target sample size.

Participants' perceptions and preferences about randomization have influenced recruitment rates in some studies (Caldwell et al., 2003; Fogg \& Gross, 2000). A number of AYA in our study declined participation because they were concerned they would not be randomized to their preferred group. In our study, some AYA preferred to be in the control audio-books group rather than in the experimental group. This finding demonstrates Piaget's tenet of the adolescent's formal operational-thinking ability to analyze a situation, such as envisioning the potential consequences of study participation according to randomization to our two planned interventions.

Additional Recruitment Strategies-Communication among study personnel was key to sustaining successful recruitment in the SMART study. Biweekly conference calls attended by all project managers and investigators provided an opportunity to discuss and monitor recruitment rates. Quarterly study reports that include recruitment rates and refusals were also helpful in fostering a dialogue within and across sites regarding recruitment trends. The reports fostered camaraderie around successes and motivated team members to be cognizant of potentially useful approaches to meeting projected recruitment rates gleaned from the literature and from other sites' recruitment experiences. For example, research team members shared their positive experiences of helping AYA learn about altruistic reasons for participating. AYA with cancer tend to have a strong sense of altruism and team members now use the phrase, "What we learn may help other kids in the future" to motivate the AYA's participation. Some participants also reported that they enjoyed being a part of a large study taking place at hospitals all over the country. The importance of health provider support as emphasized in the literature (Gooch, 2000; Knox \& Burkhart, 2007; Pletsch, Howe, \& Tenney, 1995) was also discussed by the team. As a result, our transplant physicians and HSCT nurse coordinators were further encouraged to tell potential participants of their support for the project.

\section{CONCLUSIONS AND RECOMMENDATIONS}

The success of any research study is dependent upon sufficient participant recruitment. The ability to anticipate and address recruitment challenges can make an enormous difference in the successful completion of a study. Proposed solutions to recruitment challenges as described in this article are intended to assist investigators in overcoming some of these difficulties.

When designing a study, investigators must carefully consider all factors that will influence recruitment, such as eligibility and exclusion criteria, randomization, medical practice/ referral patterns at performance sites, and general recruitment strategies. When planning for a multi-site study, we also recommend careful screening of potential study sites prior to selection as well as assessment of the support each site has from key personnel for successful recruitment. It is essential for investigators to determine if available clinical sites treat a sufficient number of patients meeting eligibility criteria or if additional clinical sites are needed to obtain the target sample. With shrinking monies available to conduct research, this factor becomes increasingly important.

Based on our findings and those in the literature, it is important to identify multiple recruitment strategies appropriate for the age and gender of the population being studied. Regardless of strategies, it is also important to note that not all recruitment strategies will be perceived as beneficial or useful to all AYA. 
Increased reporting of recruitment strategies in the literature will help to increase knowledge about the most effective approaches. We encourage investigators to report both successful and unsuccessful recruitment strategies. We also encourage journal editors to require authors to report their study's recruitment and refusal rates. Leading medical journals and international editorial groups have adopted CONSORT which suggests that documentation in the form of checklists or flow diagrams should include the number of individuals who met enrollment criteria but declined enrollment and those who were allocated to a treatment group but did not complete the study, detailing specific reasons for each scenario (Altman et al., 2001). Inclusion of this information is essential for interpreting findings and for advancing recruitment among AYA and other populations.

Researchers should seek input and support from key health care providers who care for the AYA to plan, monitor and, if necessary, adjust recruitment strategies. Academic researchers and clinical nurses (e.g., bedside nurses, nurse practitioners) must collaborate in order to recognize all factors that may influence AYA's decision-making. While each study is unique and each research team must structure, monitor, and adapt their recruitment and communication processes as needed, application of recruitment strategies used in the SMART study may augment investigators' efforts to successfully recruit AYA with cancer for future studies.

\section{References}

Altman DG, Schulz KF, Moher D, Egger M, Davidoff R, Elbourne D, et al. The revised CONSORT statement for reporting randomized trials: Explanation and elaboration. Annals of Internal Medicine. 2001; 134:663-694. [PubMed: 11304107]

Bleyer A, Budd T, Montello M. Adolescents and young adults with cancer. The scope of the problem and criticality of clincial trials. Cancer. 2006; 107(7):1645-1655. [PubMed: 16906507]

Bowman KK, Vidsten J, Kogner P, Samuelsson U. Serious illness in childhood: The different threats of cancer and diabetes from a parent perspective. The Journa of Pediatrics. 2004; 145:373-379.

Breland-Noble AM. Family first: the development of an evidence based family intervention for increasing participation in psychiatric critical care and research in depressed African American adolescents. Family Practice. 2006; 45:153-169.

Broome M, Richards D. The influence of relationships on children's and adolescent's participation in research. Nursing Research. 2003; 52:191-197. [PubMed: 12792260]

Burke ME, Albritton K, Marina N. Challenges in the recruitment of adolescents and young adults to cancer clinical trials. Cancer. 2007; 110(11):2385-2393. [PubMed: 17918260]

Caldwell PH, Butow PN, Craig JC. Parents' attitudes to children's participation in randomized controlled trials. Journal of Pediatrics. 2003; 142:554-559. [PubMed: 12756389]

Cripe, L. Early end-of-life communication map used with adults with cancer. Haase, JE., editor. Indianapolis: 2004.

Crowley R, Casarett D. Patients' willingness to participate in symptom-related and disease-modifying research. Results of a research screeening intitiative in a palliative care clinic. Cancer. 2003; 97 (9): 2327-2333. [PubMed: 12712490]

Ferrari A, Bleyer A. Tumour review: Participation of adolescents with cancer in cancer trials. Cancer Treatment Reviews. 2007; 33:603-608. [PubMed: 17250970]

Fogg L, Gross D. Focus on research methods. Threats to validity in randomized clinical trials. Research in Nursing and Health. 2000; 23:79-87. [PubMed: 10686575]

Gattuso J, Hinds PS, Tong X, Srivastava K. Methodological issues in nursing research. Monitoring child and parent refusals to enroll in clinical research. Journal of Advanced Nursing. 2006; 53 (3): 319-326. [PubMed: 16441537]

Gilligan, C., editor. In a different voice: Psychological theory and women's development. Vol. Harvard University Press; Cambridge, MA: 1982. 
Gooch W. Constructing and executing pediatric trials-The experiences of one investigator. Research Practitioner. 2000; 1:55-60.

Haase JE. The adolescent resilience model as a guide to interventions. Journal of Pediatric Oncology Nursing. 2004; 21(5):289-299. [PubMed: 15381798]

Harth SC. Parental perceptions and attitudes about informed consent in clinical research involving children. Social Science Medicine. 1995; 41:1647-1651. [PubMed: 8746864]

Hayman RM, Taylor CT, Peart NS, Galland BC, Sayers RM. Participation in research: informed consent, motivation, and influence. Journal of Pediatric Child Health. 2001; 37:51-54.

Hendricks-Ferguson VL. Relationships of age and gender to hope and spiritual well-being among adolescents with cancer. Journal of Pediatric Oncology Nursing. 2006; 23(4):189-199. [PubMed: 16766684]

Hinds PS, Oakes L, Furman W, Foppiano P, Olson MS, Quargnenti A, et al. Decision making by parents and healthcare professionals when considering continued care for pediatric patients with cancer. Oncology Nursing Forum. 1997; 24(9):1523-1528. [PubMed: 9348593]

Hinds PS, Oakes L, Furman W, Quargnenti A, Olson MS, Foppiano P. End-of-life decision making by adolescents, parents, and healthcare providers in pediatric oncology. Cancer Nursing. 2001; 24(2): 1222-1136.

Hinds PS, Oakes L, Quargnenti A, Furman W, Bowman L, Gilger E, et al. An international feasibility study of parental decision making in pediatric oncology. Oncology Nursing Forum. 2000; 27(8): 1233-1243. [PubMed: 11013904]

Hinds PS, Quargnenti A, Gattuso J, Srivastava K, Tong X, Penn L, et al. Comparing the results of coagulation tests on blood drawn by venipuncture and through tunneled venous access devices in pediatric patients with cancer. Oncology Nursing Forum. 2002; 29(3):1.

Hinds PS, Quargnenti A, Madison J. Issues in clinical nursing research: Refusal to participate in clinical nursing research. Western Journal of Nursing Research. 1995; 17(2):232-236. [PubMed: 7732689]

Hudson M, Tyc V, Srivastava K, Gattuso J, Quargnenti A, Crom D, et al. Multi-component behavioral intervention to promote health protective behaviors in childhood cancer survivors. Medical Pediatric Oncology. 2002; 39:2-11. [PubMed: 12116072]

Jones FC, Broome ME. Focus groups with African-American adolescents: Enhancing recruitment and retention in intervention studies. Journal of Pediatric Nursing. 2001; 16:88-96. [PubMed: 11326396]

Kazak AE, Alderfer MA, Streissand R, Simms S, Rourke MT, Barakat LP, et al. Treatment of postraumatic stress symptoms in adolescent survivors of childhood cancer and their families: A randomized clinical trial. Journal of Family Psychology. 2004; 18(3):493-504. [PubMed: 15382974]

Kleinpell RM. Promoting research in clinical practice. Strategies for implementing research initiatives. Advanced Critical Care. 2008; 19(2):155-163. [PubMed: 18560283]

Knox CA, Burkhart PV. Issues related to children participating in clinical research. Journal of Pediatric Nursing. 2007; 22(4):310-318. [PubMed: 17645958]

Kyngas H, Mikkonen R, Nousiainen E, Rytilahti M, Seppanen P, Vaatovaara R, et al. Coping with the onset of cancer: Coping strategies and resources of young people with cancer. European Journal of Cancer Care. 2001; 10(1):6-11. [PubMed: 11827269]

Magnusson, D.; Cairns, RB., editors. Developmental science: Toward a unified framework. New York, NY: Cambridge University Press; 1996.

Malbasa T, Kodish ED, Santacrose SJ. Adolescence adherence to oral therapy for leukemia: A focus group study. Journa of Pediatric Oncology Nursing. 2007; 24(3):139-151.

May DE, Hallin MJ, Kratochvil CJ, Puumala SE, Smith LS, Reinecke MA, et al. Factors associated with recruitement and screening in the treatment for adolescents with depression study (TADS). Journal of American Academy of Child Adolescent Psychiatry. 2007; 46(7):801-810.

Miles MS, Holditch-Davis D. Enhancing nursing research with children and families using a developmental science perspective. Annual Review of Nursing Research. 2003; 21(1):1-22. 
Pasternak RH, Geller G, Parrish C, Cheng TL. Adolescent and parent perceptions on youth participation in high risk behavior research. Archives of Pediatric Adolescent Medicine. 2007; 160:1159-1166.

Pletsch PK, Howe C, Tenney M. Recruitment of minority subjects for intervention research. Image Journal of Nursing Scholarship Fall. 1995; 27(3):211-215.

Rice, FP.; Dolgin, KG., editors. The adolescent: Development, relationships and culture. 11. Boston, MA: Pearson; 2005.

Robb SL. The effect of therapeutic music interventions on the behavior of hospitalized children in isolation: Developing a contextual support model of music therapy. Journal of Music Therapy. 2000; 37(2):118-146. [PubMed: 10932125]

Ruffin MT, Baron J. Recruitment of subjects in cancer prevention and control. Journal of Cellular Biology. 2000; 34:80-83.

Seibold-Simpson S, Morrison-Beedy D. Avoiding early study attrition in adolescent girls: Impact of recruitment contextual factors. Western Journal of Nursing Research. 2010; 32(6):761-778. [PubMed: 20634400]

Ward-Smith P, Hamlin J, Bartholomew J, Stegenga K. Quality of life among adolescents with cancer. Journal of Pediatric Oncology Nursing. 2007; 24(3):166-171. [PubMed: 17475982]

Yancey AK, Ortega AN, Kumanyika SK. Effective recruitment and retention of minority research participants. Annual of Rev Public Health. 2006; 27:1-28. 


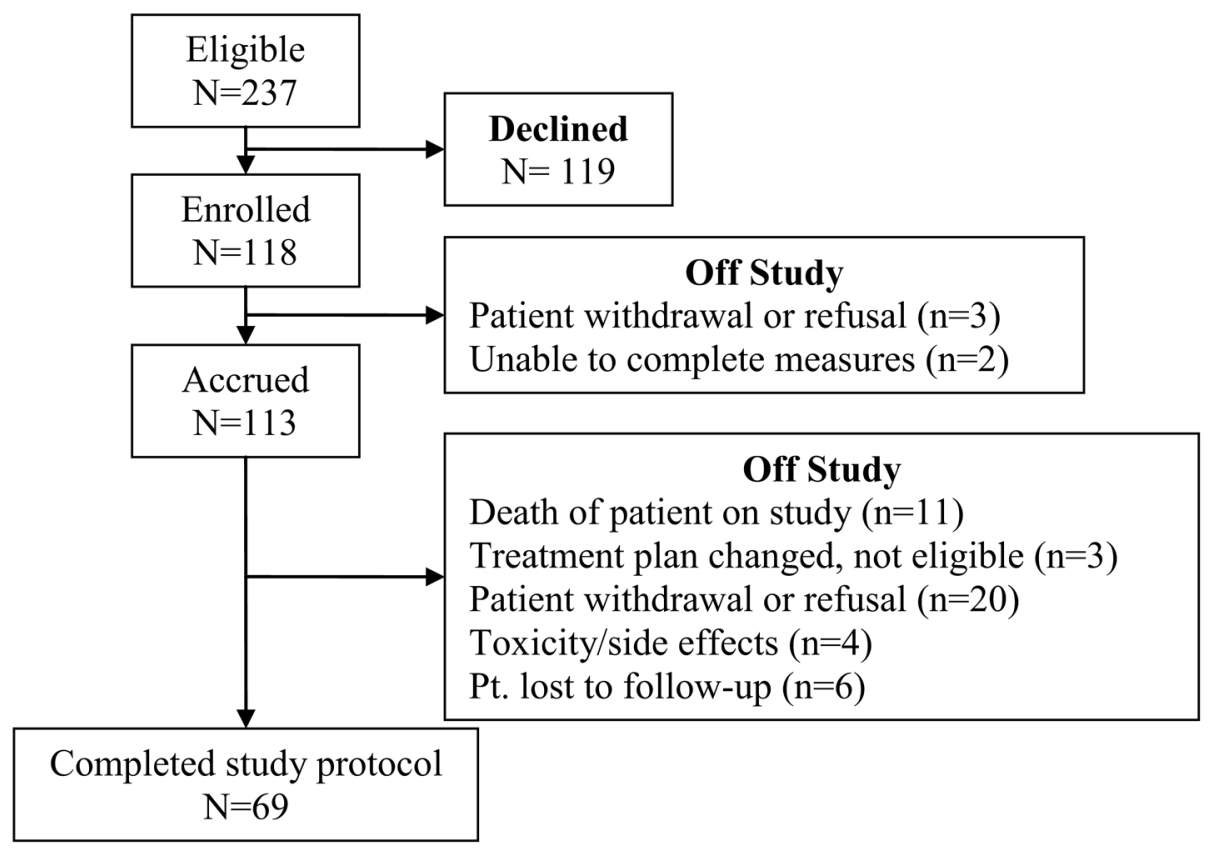

Figure 1.

CONSORT Diagram 
Table 1

Recruitment of eligible AYA by gender and age

\begin{tabular}{|c|c|c|c|c|}
\hline Eligible & Enrolled & $\begin{array}{c}\mathbf{1 1 - 1 3} \text { years Enrolled/Eligible } \\
(\boldsymbol{\%})\end{array}$ & $\begin{array}{c}\mathbf{1 4 - 1 7} \text { years Enrolled/Eligible } \\
(\boldsymbol{\%})\end{array}$ & $\begin{array}{c}\mathbf{1 8 - 2 4} \text { years Enrolled/Eligible } \\
(\boldsymbol{\%})\end{array}$ \\
\hline Female $(\mathrm{n}=83)$ & Female $(\mathrm{n}=51)$ & $16 / 24(67 \%)$ & $23 / 33(70 \%)^{*}$ & $12 / 26(46 \%)$ \\
\hline Male $(\mathrm{n}=154)$ & Male $(\mathrm{n}=67)$ & $11 / 23(48 \%)$ & $24 / 53(45 \%)$ & $32 / 77(42 \%)$ \\
\hline
\end{tabular}

* Group with highest \% enrollment: females 14-17 years of age 